\title{
Visualization of Dynamics Using Local Dynamic Modelling with Self Organizing Maps.
}

CONFERENCE PAPER in LECTURE NOTES IN COMPUTER SCIENCE · JANUARY 2007

Impact Factor: $0.51 \cdot$ Source: DBLP

CITATIONS

3

6 AUTHORS, INCLUDING:

Ignacio Díaz Blanco

University of Oviedo

44 PUBLICATIONS 128 CITATIONS

SEE PROFILE
READS

10
Juan J. Fuertes-Martínez

Universidad de León

9 PUBLICATIONS 59 CITATIONS

SEE PROFILE 


\title{
Visualization of Dynamics using Local Dynamic Modelling with Self Organizing Maps
}

\author{
Ignacio Díaz-Blanco ${ }^{1}$, Abel A. Cuadrado-Vega ${ }^{1}$, Alberto B. Diez-Gonzalez ${ }^{1}$, \\ Juan J. Fuertes-Martínez ${ }^{2}$, Manuel Domínguez-Gonzalez ${ }^{2}$, Perfecto \\ Reguera-Acevedo ${ }^{2}$ \\ 1 University of Oviedo, Area de Ingeniería de Sistemas y Automática, Campus de \\ Viesques s/n, 33204, Gijón, Asturias, Spain \\ 2 Instituto de Automática y Fabricación, Universidad de León, Escuela de \\ Ingenierías. Campus Universitario de Vegazana, León, 24071, Spain.
}

\begin{abstract}
In this work, we describe a procedure to visualize nonlinear process dynamics using a self-organizing map based local model dynamical estimator. The proposed method exploits the topology preserving nature of the resulting estimator to extract visualizations (planes) of insightful dynamical features, that allow to explore nonlinear systems whose behavior changes with the operating point. Since the visualizations are obtained from a dynamical model of the process, measures on the goodness of this estimator (such as RMSE or AIC) are also applicable as a measure of the trustfulness of the visualizations. To illustrate the application of the proposed method, an experiment to analyze the dynamics of a nonlinear system on different operating points is included.
\end{abstract}

Key words: System identification, self-organizing map, information visualization

\section{Introduction}

Information Visualization techniques have experienced a growing interest for the analysis and interpretation of large volumes of multidimensional data. Borrowed from other areas, such as bioinformatics, socioeconomics or medicine, in last years these techniques have been also used for modelling and supervision of industrial processes from data and previous knowledge. One powerful data visualization method is the self-organizing map (SOM), [6] that allows to project data in a smooth manner on a $2 \mathrm{D}$ (or $3 \mathrm{D}$ ) space that can be subject of graphical representation. This link between process data and a $2 \mathrm{D}$ space may be used to obtain 2D maps of the process states that allow to represent different features of the process in an insightful manner, helping to exploit available data and knowledge in an efficient way for process understanding [1].

However, while the SOM has been mostly used to model static relationships among the input variables, it can also be used to model nonlinear dynamical processes using local models that describe the dynamical behavior in a small region in which the process behavior can be considered approximately linear. The 
idea of assigning a dynamical model to each unit was already described in the early 90's by Kohonen as operator maps in [5], and variations of it using different training approaches were later proposed in $[9,4,2]$. One particularly efficient variant of this approach was proposed in [8] and later, with slight modifications, in [3], with excellent results, for identification and control problems in systems whose dynamics change over the operating regime.

Surprisingly, while the basic SOM has proven to be an excellent tool for exploratory data analysis, very little work has been published on using it to visualize the dynamical behavior of processes in an explicit way. In this paper, we suggest the use of the local linear modelling approach proposed in [3] introducing the notion of selectors of dynamics for the visualization of meaningful dynamical features of the local models, exploiting two major characteristics of this approach: the superior estimation accuracy of this method with respect to global approaches, that supports the accuracy of the resulting visualizations, and the topological order of the resulting local models -which occurs under certain mild hypotheses- that ensures a proper visualization.

\section{Local linear modelling of dynamics}

Let's consider the following parametric Nonlinear AutoRegresive with eXogenous inputs (p-NARX) system that express the present output as a function of past outputs and inputs as well as of a set of parameters.

$$
y(k)=f\left(y(k-1), \ldots, y\left(k-n_{y}\right), u(k), \ldots, u\left(k-n_{u}\right), p_{1}(k), \ldots, p_{p}(k)\right)
$$

This model can be expressed in a more compact way as

$$
y(k)=f(\varphi(k), \mathbf{p}(k))
$$

where $\varphi(k)=[y(k-1), \cdots, y(k-n), u(k), \cdots, u(k-m)]^{T}$ is a vector of known data at sample $k, \mathbf{p}=\left[p_{1}(k), \cdots, p_{p}(k)\right]^{T}$ is a vector of parameters, and $f(\cdot, \cdot)$ is a given functional relationship that may be linear or nonlinear. Model (2) describes a dynamic relationship between the process inputs and outputs determined by the values of $p_{1}(k), \ldots, p_{p}(k)$.

\subsection{Clustering dynamics}

Let's consider a set of available process variables $\mathbf{s}(k)=\left[s_{1}(k), \cdots, s_{s}(k)\right]^{T} \in \mathcal{S}$ that are known to discriminate different dynamical process behaviors, that is, such that $\mathbf{p}(i) \neq \mathbf{p}(j) \Longrightarrow \mathbf{s}(i) \neq \mathbf{s}(j)$; under this hypothesis, these variables can be regarded as selectors of dynamics, since information regarding the dynamic relationship between the process' inputs and outputs is preserved in the selectors.

In a first stage, a SOM with $N$ units is trained in the selectors space $\mathcal{S}$ to cluster the process dynamics. Each unit $i$ is associated to a $s$-dimensional prototype vector $\mathbf{m}_{i}$ in $\mathcal{S}$ and a position vector on a low dimensional regular 
grid, $\mathbf{g}_{i}$, in the output space. For each vector $\mathbf{s}(k)$ the best matching unit is computed as

$$
c(k)=\arg \min _{i}\left\{\mathbf{s}(k)-\mathbf{m}_{i}(t)\right\}
$$

Then, an adaptation stage is performed according to

$$
\mathbf{m}_{i}(t+1)=\mathbf{m}_{i}(t)+\alpha(t) h(c(k), i)\left[\mathbf{s}(k)-\mathbf{m}_{i}(t)\right]
$$

where $\alpha(t)$ is the learning rate and $h(.,$.$) is the neighborhood function. After$ convergence, the result is a set of prototype vectors that divide $\mathcal{S}$ into a finite set of Voronoi regions each of which defines a different dynamic behavior.

\subsection{Local model estimation}

Once a SOM has been trained with the $\{\mathbf{s}(k)\}$, a model $i$ may be estimated for each prototype $\mathbf{m}_{i}$ using all the pairs $\{y(k), \varphi(k)\}$ such that

$$
\|\mathbf{g}(c(k))-\mathbf{g}(i)\| \leq \sigma_{\text {loc }} \quad \text { where } \quad c(k)=\arg \min _{i}\left\{\mathbf{s}(k)-\mathbf{m}_{i}(t)\right\}
$$

that is, those pairs whose corresponding selectors $\mathbf{s}(k)$ are mapped onto a neighborhood of $\mathbf{m}_{i}$ of width $\sigma_{\text {loc }}$. A particular choice can be a linear model

$$
y(k)=\mathbf{p}_{i}^{T} \varphi(k)+\varepsilon(k), \quad \text { for neuron } i
$$

whose parameters $\mathbf{p}_{i}$ can be obtained using least squares to fit the aforementioned pairs $\{y(k), \varphi(k)\}$. This corresponds to a local ARX model associated to neuron $i$, that can be rewritten using the difference equation notation

$$
y(k)=\sum_{j=1}^{n_{y}} a_{j}^{i} y(k-j)+\sum_{j=0}^{n_{u}} b_{j}^{i} u(k-j)+\varepsilon(k)
$$

or as a transfer function

$$
G\left(z, \mathbf{p}_{i}\right)=\frac{Y(z)}{U(z)}=\frac{\sum_{j=0}^{n_{u}} b_{j}^{i} z^{-j}}{1-\sum_{j=1}^{n_{y}} a_{j}^{i} z^{-j}}
$$

being $\mathbf{p}_{i}=\left[a_{1}^{i}, \ldots, a_{n_{y}}^{i}, b_{0}^{i}, b_{1}^{i}, \ldots, b_{n_{u}}^{i}\right]$

\subsection{Retrieval}

Once the model is trained, a local model $f\left(\varphi(k), \mathbf{p}_{i}\right)$ is assigned to each neuron $i$. The problem of retrieval is stated as to get $y(k)$ given $\varphi(k)$ and the dynamic selectors $\mathbf{s}(k)$. This is accomplished in two steps: 1) obtain the best matching unit, $c(k)=\arg \min _{i}\left\{\mathbf{s}(k)-\mathbf{m}_{i}(t)\right\}$ and 2) apply the local model $y(k)=f\left(\varphi(k), \mathbf{p}_{c(k)}\right)$. Note that depending on the problem (one or multiple step ahead prediction) data vector $\varphi(k)$ may contain real or estimated past outputs. 


\section{Visualization of dynamics}

\subsection{Election of the dynamic selectors}

After the steps outlined in sections 2.1 and 2.2, the result is a set of prototype vectors $\mathbf{m}_{i}$ in the space of dynamic selectors, along with companion vectors $\mathbf{p}_{i}$ containing the model parameters, $\left\{\mathbf{m}_{i}, \mathbf{p}_{i}\right\}$.

A key issue for visualization is topology preservation. Since the prototype vectors $\mathbf{m}_{i}$ have been directly obtained from the SOM algorithm (3),(4), they may be expected to be properly ordered for visualization as largely seen in the literature $[6,7]$. But, what about the $\mathbf{p}_{i}$ ?. Under the hypothesis of discriminating dynamic selectors stated in sec. 2.1 -i.e., different dynamical behaviors never occur for the same values of the dynamic selectors- and requiring a somewhat smooth relationship between the selectors and the process dynamics, the SOM topology preservation will maintain this smoothness on the maps by transitivity. In other words, smooth transitions between the dynamics of nearby prototypes will emerge. This means that not only component planes obtained from prototype vectors $\mathbf{m}_{i}$ but also planes of the elements of $\mathbf{p}_{i}$, or physically insightful transformations of them, will be smooth and susceptible to be visualized.

Despite these apparently restrictive conditions a proper choice of the dynamic selectors can be often done from problem domain knowledge. This is often the case for nonlinear processes whose local dynamics at sample $k$, (say, $\mathbf{p}(k)$ ) depend on the current working point (say, $\mathbf{s}(k)$ ). Also, proper choices can be made with little prior knowledge inspired on the Takens embedding theorem, as suggested in [8] and [3], such as using an embedded vector with delayed versions of one or more process outputs as dynamic selectors

$$
\mathbf{s}(k)=[y(k-1), y(k-2), \ldots, y(k-q)]^{T}
$$

Note however, that the dynamic selectors do not need to be restricted to the embedding space of the outputs as suggested in [3]. Indeed, other process variables -specially those suspected to define working points with different dynamics- may be included in $\mathbf{s}(k)$ to evaluate their influence on the process dynamics by means of the SOM visualizations.

\subsection{Visualization of dynamic features}

The parameter vectors $\mathbf{p}_{i}$ convey information about the process dynamics. As seen in eqs. (7) and (8), in the linear case the $\mathbf{p}_{i}$ is equivalent to a difference equation or a transfer function. A trivial approach to visualize the process dynamics is to visualize the component planes of the elements of $\mathbf{p}_{i}$. Each component plane [6] is obtained by assigning to each node $\mathbf{g}_{i}$ in the SOM grid a gray or color level proportional to the scalar values of the elements $a_{j}^{i}$ and $b_{j}^{i}$ of $\mathbf{p}_{i}$ associated to node $i$. To get some insight on the dynamics, however, there are other possible representations that can be expressed as parametric transformations 
$T\left(\mathbf{p}_{i}, \theta_{1}, \theta_{2}, \ldots\right)$ of the elements of $\mathbf{p}_{i}$. A useful representation, for instance, is the power density of a transfer function at normalized frequency $\theta$

$$
T\left(\mathbf{p}_{i}, \theta\right)=\left|\frac{\sum_{j=0}^{n_{u}} b_{j}^{i} e^{-j \theta}}{1-\sum_{j=1}^{n_{y}} a_{j}^{i} e^{-j \theta}}\right|^{2}
$$

Of course any other straightforward transformations in the same idea of (10) are suitable such as peak gains, resonant frequencies, bandwidth, etc.

\section{Experimental results}

The proposed method was tested on simulated data of a nonlinear system consisting of a pendulum of mass $m$ and length $l$ mounted on a car whose basis is subject to an acceleration $u$ (see fig. 1).
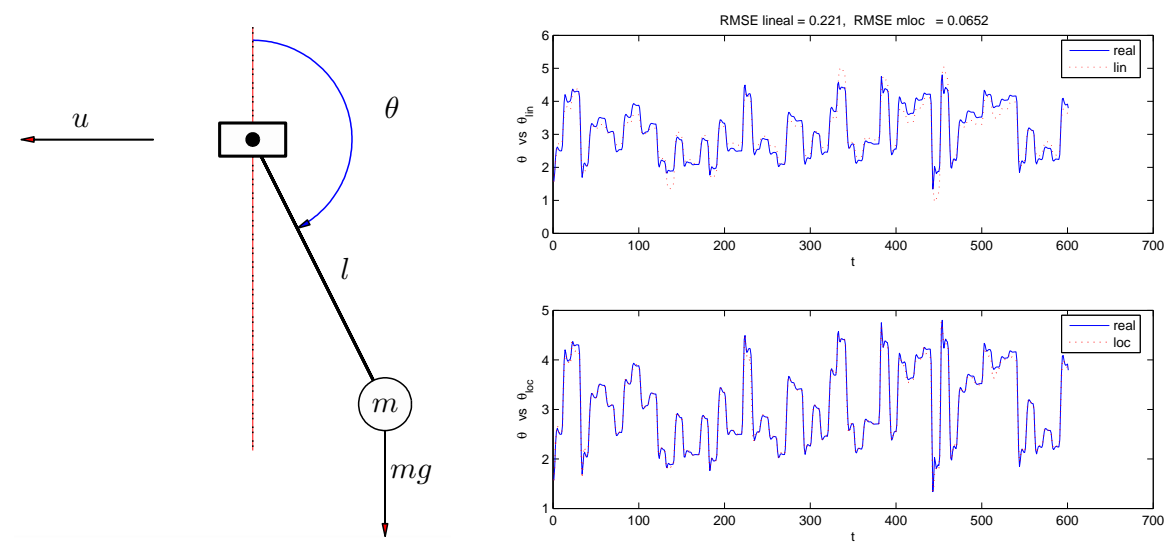

Fig. 1. On the left, the schematics of the pendulum; on the right, comparison of global linear model against local models on test data.

$$
J \ddot{\theta}+B \dot{\theta}=m g l \sin \theta+m l u \cos \theta
$$

where $J$ is the moment of inertia w.r.t. the pivot, $B$ is the friction coefficient and $g=9.8 \mathrm{~m} / \mathrm{s}^{2}$ is gravity acceleration. Choosing $\mathbf{x}=\left[x_{1}, x_{2}\right]^{T}=[\theta, \dot{\theta}]^{T}$, and $y=\theta$, it can be expressed in state space form

$$
\begin{aligned}
\dot{x}_{1} & =x_{2} \\
\dot{x}_{2} & =\frac{1}{J}\left(m g l \sin x_{1}+m l u \cos x_{1}-B x_{2}\right) \\
y & =x_{1}
\end{aligned}
$$


that reveals a nonlinear state space dynamics of the type $\dot{\mathbf{x}}=f(\mathbf{x}, u)$ showing different local behaviors on different state space regions. The linearized model for small movements around an equilibrium point defined by $\theta_{0}$, in transfer function form is

$$
G\left(s, \theta_{0}\right)=\frac{m l \cos \theta_{0}}{J s^{2}+B s-m g l \cos \theta_{0}}
$$

that, as seen, depends on $\theta_{0}$. This system is nonlinear and exhibits different local dynamic behaviors depending on the working point defined by $\theta$. An embedded vector with delayed versions of the output, as shown in eq. (9), was selected as dynamic selectors, as suggested in [8]. As in [3], good results were achieved with low values of $q$, and we finally selected $q=2$ to yield

$$
\mathbf{s}(k)=[\theta(k-1), \theta(k-2)]^{T}
$$

Conceptually, this allows to take into account both the current angle and speed of the pendulum, $[\theta, \dot{\theta}]^{T}$, since both states can be derived through linear transformations of the elements of $\mathbf{s}(k)$ as $\theta \approx \theta(k-1)$ and $\dot{\theta} \approx \frac{\theta(k-1)-\theta(k-2)}{\Delta t}$

To evaluate the proposed method, the system was simulated under random steps of the car acceleration $u$ of 5 seconds duration each, with a sample time $t_{s}=0.5 \mathrm{sec}$. and a total simulation time of $5000 \mathrm{sec}$., resulting in vectors $\theta(k)$ and $u(k)$ of 10000 samples. A $20 \times 20$ SOM was trained using the batch algorithm on vectors $\mathbf{s}(k)=[\theta(k-1), \theta(k-2)]^{T}$ with a PCA normalization and a gaussian neighborhood $h_{i j}(t)=e^{-\frac{d(i, j)}{\sigma(t)^{2}}}$ where $\sigma(t)$ was made to decrease monotonically from 7 to 0.3 .

Once the SOM was trained, local ARX models in the form (7) were trained for all combination of orders $n_{y}=\{1,2,3\}$ and $n_{u}=\{1,2,3\}$ and using local model widths $\sigma_{\text {loc }}=\{2,3\}$ in eq. (5). For each pair $\left(n_{y}, n_{u}\right)$ the model was evaluated against validation data, and the Akaike's information criterion (AIC)

$$
A I C=\log \left(\sum_{i=1}^{N}[y(i)-\hat{y}(i)]^{2}\right)+2 d / N
$$

was computed for all the local models to select the best model order, where $d=$ $n_{y}+n_{u}+2$ is the total number of model parameters including a bias term, and $N$ is the number of estimation data. From table 1 the best performance is achieved by the $10^{\text {th }}$ model type consisting of $\operatorname{ARX}(2,2)$ using a local model width $\sigma_{\text {loc }}=$ 3. The prediction ability of this model on test data is compared to the global linear model in fig. 1, showing that it clearly outperforms the global linear model. This suggests that information regarding the system's dynamical behavior is present in the dynamical model based on the local approach. Since the local models are topologically ordered by the SOM, meaningful properties for single local ARX models (such as e.g. spectral densities at given frequencies) can be visualized for all the models by means of SOM maps, providing a comprehensive description of all the dynamic modes of the nonlinear system -see fig. 2. 


\begin{tabular}{r|rrrrrr} 
& $n_{u}$ & $n_{y}$ & $\sigma_{\text {loc }}$ & RMSE (loc) & RMSE (lin) & AIC (loc) \\
\hline 1 & 1 & 1 & 2 & 0.163 & 0.252 & -3.59 \\
2 & 1 & 1 & 3 & 0.143 & 0.252 & -3.84 \\
3 & 1 & 2 & 2 & 0.168 & 0.328 & -3.52 \\
4 & 1 & 2 & 3 & 0.145 & 0.328 & -3.81 \\
5 & 1 & 3 & 2 & 1.18 & 0.532 & 0.396 \\
6 & 1 & 3 & 3 & 0.231 & 0.532 & -2.87 \\
7 & 2 & 1 & 2 & 0.0557 & 0.222 & -5.72 \\
8 & 2 & 1 & 3 & 0.06 & 0.222 & -5.58 \\
9 & 2 & 2 & 2 & 0.0545 & 0.223 & -5.76 \\
10 & 2 & 2 & 3 & 0.0528 & 0.223 & -5.86 \\
11 & 2 & 3 & 2 & 18.8 & 0.493 & 5.94 \\
12 & 2 & 3 & 3 & 0.534 & 0.493 & -1.18 \\
13 & 3 & 1 & 2 & 0.0528 & 0.235 & -5.82 \\
14 & 3 & 1 & 3 & 0.0645 & 0.235 & -5.46 \\
15 & 3 & 2 & 2 & 0.108 & 0.26 & -4.38 \\
16 & 3 & 2 & 3 & 0.451 & 0.26 & -1.57 \\
17 & 3 & 3 & 2 & 1.18 & 0.257 & 0.418 \\
18 & 3 & 3 & 3 & 100 & 0.257 & 100
\end{tabular}

Table 1. Comparison of different model orders against validation data.

As a matter of fact, the dc gain can be computed at $s=0$ in the linearized model (15) for all equilibrium points as $G(0)=-1 / g$, regardless the value of the equilibrium point $\theta_{0}$. However, in the nonlinear system, the angle $\theta$ for a constant acceleration $u_{0}$ can be shown to be $\theta_{0}=\arctan \left(-u_{0} / g\right)$ at steady state, that is, the dc gain is $\frac{\arctan \left(-u_{0} / g\right)}{u_{0}}$, that approximately coincides with $-1 / g$ for small values of $u_{0}$, near the working point $\theta_{0}=\pi$. This real behavior is shown in the frequency map for $\omega=0$ (dc gain), where the dc gain of the system is approximately $-20 \mathrm{~dB}(=0.1 \approx 1 / g)$ for values of $\theta$ around $\pi$, but decreases as the pendulum approaches to the horizontal positions $\theta$ around $\pi / 2$ or $3 \pi / 2$.

Finally, other more subtle dynamical relationships can also be observed, such as the relationship between map of angular speed $(\Delta y(k-1) \stackrel{\text { def }}{=} y(k-1)-y(k-2))$ and the harmonics at $0.3 f_{s}$ and $0.35 f_{s}$, for which it seems that the nonlinear model of the pendulum exhibits surprisingly lower gains for large angular speeds regardless the sign of this speed.

\section{Conclusions}

In this work we have proposed a method to visualize the local dynamical behaviors of nonlinear processes using the SOM local linear approach described in [3] with some assumptions that require some prior knowledge -usually available from problem domain- about the variables that can have an influence on the process dynamics. Since the visualizations are derived from a dynamical model used for estimation, the accuracy of the visual descriptions of dynamics may be measurable, in some sense, using standard performance indices (such as RMSE 

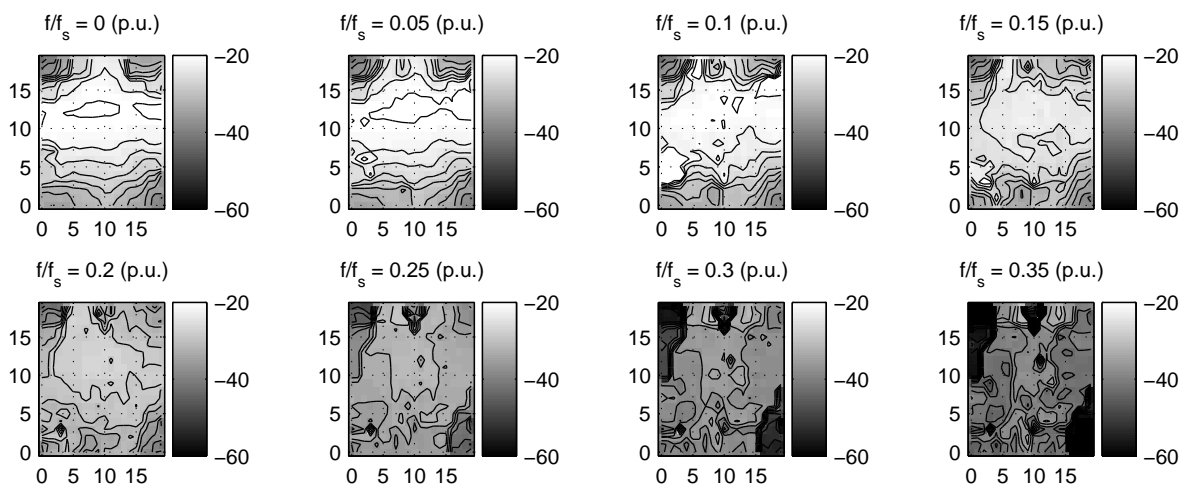

$$
f / f=0.25 \text { (p.u.) }
$$

$\mathrm{f} / \mathrm{f}_{\mathrm{s}}=0.3$ (p.u.)
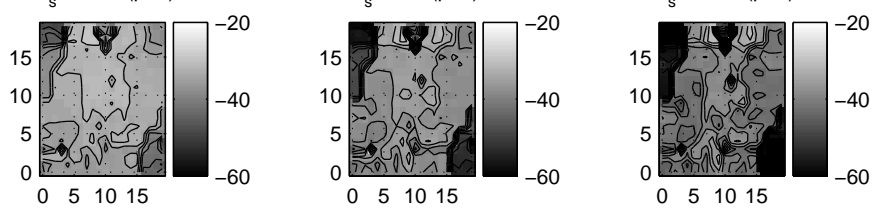

$\mathrm{f} / \mathrm{f}=0.4$ (p.u.)

$\mathrm{f} / \mathrm{f}_{\mathrm{s}}=0.45$ (p.u.)
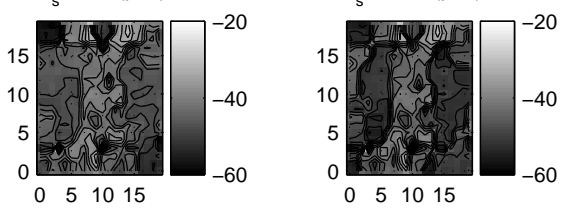

$f / f=0.5$ (p.u.)

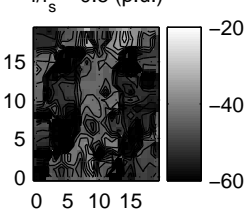

$\mathrm{f} / \mathrm{f}_{\mathrm{s}}=0.55$ (p.u.)

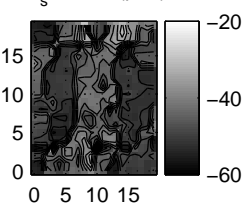

$\mathrm{y}(\mathrm{k}-1)$

$y(k-2)$
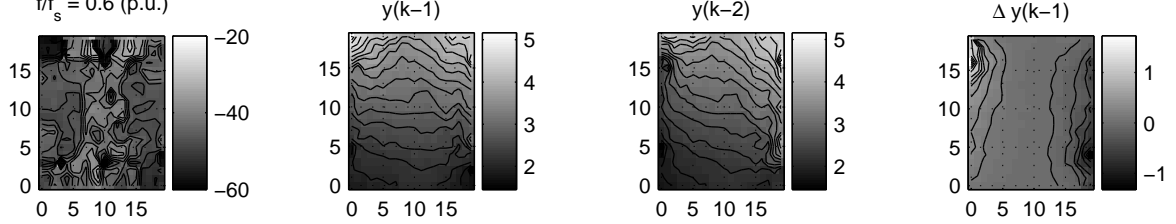

Fig. 2. Frequency response maps. Frequencies are expressed in p.u. with respect to the sample rate $f_{s}$; gains at each frequency are expressed in $\mathrm{dB}$.

or AIC) of the estimator on test data. Experimental results with simulated data of a nonlinear system are included to show its application.

While the proposed method has proven to work well under reasonable conditions in other problems, some unsolved questions still remain, such as the requirable conditions for adequate persistence of excitation of the training data, as well as the interpretability of deficient rank or locally unstable models, that may arise even for a good accuracy of the underlying estimator.

Acknowledgement. This research has been financed by the Spanish Ministerio de Educación y Ciencia under grant DPI2006-13477-C02-01.

\section{References}

1. Esa Alhoniemi, Jaakko Hollmén, Olli Simula, and Juha Vesanto. Process monitoring and modeling using the self-organizing map. Integrated Computer Aided Engineering, 6(1):3-14, 1999 . 
2. GA Barreto and AFR Araujo. Identification and control of dynamical systems using the self-organizing map. Neural Networks, IEEE Transactions on, 15(5):1244-1259, 2004.

3. Jeongho Cho, Jose C. Principe, Deniz Erdogmus, and Mark A. Motter. Modeling and inverse controller design for an unmanned aerial vehicle based on the selforganizing map. IEEE Transactions on Neural Networks, 17(2):445-460, March 2006.

4. O. Fontenla-Romero, A. Alonso-Betanzos, E. Castillo, J.C. Principe, and B. Guijarro-Berdinas. Local Modeling Using Self-Organizing Maps and Single Layer Neural Networks. Proceedings of the International Conference on Artificial Neural Networks, pages 945-950, 2002.

5. T. Kohonen. Generalizations of the self-organizing map. Proceedings of 1993 International Joint Conference on Neural Networks, 1993. IJCNN'93-Nagoya, 1:457-462, 1993.

6. Teuvo Kohonen. The self-organizing map. Proceedings of the IEEE, 78(9):14641480, september 1990.

7. Teuvo Kohonen, Erkki Oja, Olli Simula, Ari Visa, and Jari Kangas. Engineering applications of the self-organizing map. Proceedings of the IEEE, 84(10):1358-1384, october 1996.

8. Jose C. Principe, Ludong Wang, and Mark A. Motter. Local dynamic modeling with self-organizing maps and applications to nonlinear system identification and control. Proceedings of the IEEE, 86(11):2240-2258, Nov 1998.

9. Juha Vesanto. Using the SOM and local models in time-series prediction. In Proceedings of the Workshop Self-Organizing Maps (WSOM), pages 209-214, Helsinki University of Technology, Finland, June 4-6, May 1997. 